\title{
Prevalence and phylogenetic analysis of porcine deltacoronavirus in Sichuan province, China
}

\author{
Yu Feng ${ }^{1} \cdot$ Zhiwen $X u^{1,2}(1) \cdot$ Ling $Z u^{1,2}$
}

Received: 23 October 2019 / Accepted: 4 August 2020 / Published online: 6 September 2020

(c) Springer-Verlag GmbH Austria, part of Springer Nature 2020

\begin{abstract}
In order to understand the prevalence and genetic diversity of porcine deltacoronavirus (PDCoV) in diarrhoeal pigs in Sichuan province, 634 clinical samples were collected from individual pigs with diarrhoea in 13 regions of Sichuan province, China, from January 2017 and June 2019. The detection results showed that the infection rate of PDCoV was relatively low in diarrhoeal pigs, $13.25 \%$ (84/634), but the infection rate of PEDV (porcine epidemic diarrhea virus) was high, 32.18\% (204/634). Coinfection with PEDV was common (55.95\%, 47/84) in PDCoV-infected diarrhoeal pigs. Additionally, the chance of PDCoV infection was 2.77 times higher in suckling piglets than in sows, and about 3.30 times higher in spring and winter than in summer. PDCoV/PEDV coinfection was $75 \%$ less likely in sows than in suckling piglets. The complete genomes of four Sichuan PDCoV strains were sequenced and analysed. There were some insertion-deletion signatures in the whole genome sequences of four strains, including a 6-nt deletion in the non-structural gene 2 region, a 9-nt insertion in the non-structural gene 3 region, a 3-nt deletion in the S gene region, and a distinguishing 11-nt deletion in the 3'UTR region. Phylogenetic analysis based on complete genome sequences revealed that the PDCoV Sichuan strains were closely related to other Chinese PDCoV reference strains; however, phylogenetic analysis based on $\mathrm{S}$ gene sequences showed that the $\mathrm{CH} /$ SC/2019 strain clustered in a large clade with strains from the USA, Japan, and Korea. These data advance our understanding of the genetic diversity and evolutionary characteristics of PDCoV in China and may contribute to vaccine development.
\end{abstract}

\section{Introduction}

Porcine deltacoronavirus (PDCoV), a member of the genus Deltacoronavirus, is a single-stranded, positive-sense enveloped RNA virus that causes enteritis and watery diarrhoea in pigs, especially nursing piglets [1]. The full genome of $\mathrm{PDCoV}$ is approximately $25.4 \mathrm{~kb}$ long, which is the smallest genome size among the porcine coronaviruses, arranged in the order $5^{\prime}$ untranslated region (UTR), open reading frame 1a and b (ORF1ab), spike (S), envelope (E), membrane (M), non-structural gene 6 (NS6), nucleocapsid (N), nonstructural gene 7 (NS7), and 3'UTR [2]. The PDCoV S gene is frequently used for studying genetic relationships among

Handling Editor: Tim Skern.

Zhiwen $\mathrm{Xu}$

abtcxzw@126.com

1 College of Veterinary Medicine, Sichuan Agricultural University, No. 211 Huiming Road, Chengdu 611130, China

2 Animal Biology Technology Center, Sichuan Agricultural University, Chengdu, China different PDCoV strains and for performing epidemiological investigations [3].

PDCoV was first discovered in Hong Kong, and has since been reported in many countries, including the USA, Canada, Korea, China, Thailand, Laos, and Vietnam [4-9]. In China, PDCoV had been reported in many provinces, but little has been reported regarding the prevalence and epidemiology of PDCoV in Sichuan province, a major pig-raising province of China. Thus, in this study, we investigated the prevalence of PDCoV in diarrhoeal pigs and analysed the complete genome sequences of PDCoV isolates from Sichuan, China.

\section{Materials and methods}

\section{Sampling}

In this study, a total of 634 intestinal and faecal clinical samples (405 intestinal samples and 229 faecal samples) were collected from pigs with diarrhoea outbreaks diagnosed by a pig farm veterinarian from January 2017 and June 2019. All 
samples were collected from suckling piglets and sows and from pig farm in 13 regions in Sichuan province. The sampling locations are shown in Table 1. Soon after sampling, faeces and intestinal contents were prepared as $10 \%$ suspensions in phosphate-buffered saline (PBS). The suspensions were then vortexed and centrifuged at $8000 \mathrm{~g}$ at $4^{\circ} \mathrm{C}$ for 10 min. The clarified supernatants were stored at $-80^{\circ} \mathrm{C}$ until use for RNA extraction. Total RNAs were extracted using TRIzol Reagent (Invitrogen, USA) according to the manufacturer's instructions. RT-qPCR was carried out to assess the frequency of PDCoV monoinfections and coinfections with PEDV and transmissible gastroenteritis virus (TGEV). We used the virotype PEDV/TGEV/PDCoV RT-PCR Rgt (QIAGEN) following the manufacturer's protocol.

\section{Complete genome sequences and phylogenetic analysis}

Representative PDCoV strains from four different pig farms where severe or moderate diarrhoea had been reported were selected for sequencing of the complete genome by RT-PCR amplification of 24 regions covering the PDCoV genome as described previously [10]. These representative PDCoV strains were named CH/SC/2017, CH/SC/2018/1, $\mathrm{CH} / \mathrm{SC} / 2018$, and $\mathrm{CH} / \mathrm{SC} / 2019$ (GenBank accession nos. MK211169, MK005882, MK330605, and MK993519). The PCR products were purified and cloned into the vector pMD18-T. Positive clones of each amplicon was sequenced twice (Sangon Biotech, Shanghai, China). At least three independent PCR amplicons were sequenced to obtain a consensus sequence. Complete sequences of four PDCoV strains were assembled by combining overlapping contigs and trimming off primer sequences using the DNASTAR software package (DNASTAR Inc., Madison, WI, USA). Sequence alignment analysis of these four strains and other PDCoV strains with sequences in the GenBank database was conducted using the Megalign program. Phylogenetic trees based on complete genome and $\mathrm{S}$ gene sequences were constructed by the maximum-likelihood method using MEGA software version 6 with bootstrap analysis of 1,000 replicates.

\section{Statistical analysis}

The rates of infection with PDCoV and coinfection with PDCoV and PEDV in diarrhoeal pigs were analysed according to age, year, and season. Significance was set at $p<0.05$. Possible risk factors associated with virus infection were analysed by univariate and multivariate exact logistic regression models using Stata/SE 15.1 (Stata Corporation, USA). For each model the odds ratio (OR) and 95\% confidence interval (CI) were calculated.

\section{Results}

\section{Prevalence of PDCoV}

Of the 634 clinical samples tested in our PDCoV prevalence survey, 84 (13.25\%) were positive for PDCoV and 204 (32.18\%) were PEDV positive. The positive rate for PDCoV in sows and piglets was 6.80\% (14/206) and 16.36\% (70/428), respectively. All of the samples were negative for TGEV, as shown in Table 2. The results indicate that the prevalence rate of PDCoV was relatively low in diarrhoeal pigs in Sichuan and that PEDV was the main pathogen causing diarrhoea in pigs. It is also noteworthy that 47 samples that were positive for PDCoV were also positive for PEDV, showing that mixed infections with these viruses are relatively common. The PDCoV infection rates in different regions of Sichuan province are shown in Table 1. Twelve out of the 13 regions were considered PDCoV positive, including the regions of Mianyang (14.93\%), Deyang (16.67\%), and Guanan (22.73\%), suggesting that PDCoV is widespread on pig farms in Sichuan province.

As shown in Table 3, The results show that the prevalence of PDCoV in diarrhoeal pigs increased from $10.53 \%$ in 2017 to $17.24 \%$ in 2019; however, based on statistical analysis, we could not conclude that PDCoV infection in diarrhoeal pigs has been increasing from year to year $(p$ $>0.05$ ). More clinical samples are needed to determine whether the PDCoV infection rate is correlated with the
Table 1 The number of samples and PDCoV positive rates in different regions in Sichuan, China

\begin{tabular}{llllll}
\hline Region & $\begin{array}{l}\text { Number of } \\
\text { samples }\end{array}$ & PDCoV positive (\%) & Region & $\begin{array}{l}\text { Number of } \\
\text { samples }\end{array}$ & PDCoV positive (\%) \\
\hline Mian Yang & 67 & $10(14.93 \%)$ & Ya An & 55 & $5(9.09 \%)$ \\
Guan Yuan & 30 & $4(13.33 \%)$ & De Yang & 48 & $8(16.67 \%)$ \\
Guan An & 44 & $10(22.73 \%)$ & Nan Chong & 93 & $13(13.98 \%)$ \\
Sui Ning & 23 & $2(8.70 \%)$ & Zi Yang & 50 & $8(16.00 \%)$ \\
Mei Shan & 49 & $6(12.24 \%)$ & Da Zhou & 41 & $7(17.07 \%)$ \\
Le Shan & 31 & $0(0 \%)$ & Nei Jiang & 25 & $3(12.00 \%)$ \\
Yi Bing & 78 & $8(10.26 \%)$ & Total & 634 & $84(13.25 \%)$ \\
\hline
\end{tabular}


Table 2 Detection of PDCoV, PEDV, and TGEV in diarrhoeal samples from pigs in Sichuan, China, 2017-2019

\begin{tabular}{|c|c|c|c|c|c|c|c|c|c|c|}
\hline \multirow[b]{2}{*}{ Year } & \multirow[b]{2}{*}{ Virus } & \multicolumn{3}{|l|}{ Sows } & \multicolumn{3}{|c|}{ Suckling piglets } & \multicolumn{3}{|l|}{ Total } \\
\hline & & $\begin{array}{l}\text { Number } \\
\text { of sam- } \\
\text { ples }\end{array}$ & Positive & Positive rate, $\%$ & $\begin{array}{l}\text { Number } \\
\text { of sam- } \\
\text { ples }\end{array}$ & Positive & Positive rate, $\%$ & $\begin{array}{l}\text { Number } \\
\text { of sam- } \\
\text { ples }\end{array}$ & Positive & Positive rate, $\%$ \\
\hline \multirow{6}{*}{2017} & PDCoV & 76 & 5 & 6.58 & 152 & 19 & 12.50 & 228 & 24 & 10.53 \\
\hline & PEDV & & 17 & 22.37 & & 53 & 34.87 & & 70 & 30.70 \\
\hline & TGEV & & 0 & 0 & & 0 & 0 & & 0 & 0 \\
\hline & $\mathrm{PDCoV}+\mathrm{PEDV}$ & & 1 & 1.32 & & 13 & 8.55 & & 14 & 6.14 \\
\hline & PDCoV & 94 & 6 & 6.38 & 196 & 34 & 17.35 & 290 & 40 & 13.79 \\
\hline & PEDV & & 21 & 22.34 & & 71 & 36.22 & & 92 & 31.72 \\
\hline \multirow[t]{4}{*}{2018} & TGEV & & 0 & 0 & & 0 & 0 & & 0 & 0 \\
\hline & PDCoV+PEDV & & 3 & 3.19 & & 23 & 11.73 & & 26 & 8.97 \\
\hline & PDCoV & 36 & 3 & 8.33 & 80 & 17 & 21.25 & 116 & 20 & 17.24 \\
\hline & PEDV & & 10 & 27.78 & & 32 & 40.00 & & 42 & 36.21 \\
\hline \multirow[t]{3}{*}{2019} & TGEV & & 0 & 0 & & 0 & 0 & & 0 & 0 \\
\hline & PDCoV+PEDV & & 0 & 0 & & 7 & 8.75 & & 7 & 6.03 \\
\hline & PDCoV & 206 & 14 & 6.80 & 428 & 70 & 16.36 & 634 & 84 & 13.25 \\
\hline $2017-$ & PEDV & & 48 & 23.30 & & 156 & 36.45 & & 204 & 32.18 \\
\hline \multirow[t]{2}{*}{2019} & TGEV & & 0 & 0 & & 0 & 0 & & 0 & 0 \\
\hline & PDCoV+PEDV & & 4 & 1.94 & & 43 & 10.05 & & 47 & 7.41 \\
\hline
\end{tabular}

Table 3 Analysis of univariate and multivariate exact logistic regression models of possible risk factors associated with PDCoV and PDCoV-PEDV coinfection

\begin{tabular}{lllll}
\hline Variable & & OR & $95 \%$ CI & $p$-value \\
\hline $\begin{array}{llll}\text { Univariate } \\
\text { Age }\end{array}$ & & & & \\
& Sow & 1 & ref & \\
Year & Piglet & 2.77 & $1.52-5.04$ & 0.001 \\
& & & & \\
& 2017 & 1 & ref & \\
Season & 2018 & 1.36 & $0.79-2.33$ & 0.263 \\
& 2019 & 1.77 & $0.93-3.36$ & 0.081 \\
& & & & \\
& Summer & 1 & ref & \\
& Spring & 3.30 & $1.12-9.65$ & 0.030 \\
& Autumn & 1.85 & $0.57-5.94$ & 0.304 \\
& Winter & 3.32 & $1.19-9.13$ & 0.022 \\
Multiple model & & & & \\
& Age of piglet & 2.67 & $1.46-4.87$ & 0.001 \\
& Spring season & 3.24 & $1.32-8.78$ & 0.028 \\
& Winter season & 3.30 & $1.40-9.08$ & 0.022 \\
Coinfection multivariate model & & & \\
& Age of piglet & 3.98 & $1.14-13.97$ & 0.031 \\
\hline
\end{tabular}

ref, reference variable; OR, odds radio; $\mathrm{CI}$, confidence interval

year of sample collection. Moreover, analysis of individual variables revealed that suckling piglets were 2.77 (OR) times more likely to be infected than sows. Additionally, the PDCoV positive rates in spring $(15.46 \%, 32 / 207)$ and winter $(16.22 \%, 36 / 222)$ were clearly higher than in summer $(5.26 \%, 4 / 76)$. Pigs had about 3.30 (OR) times more risk of PDCoV infection in spring and winter than in summer. PDCoV/PEDV coinfection was statistically correlated with age. Coinfection was $75 \%$ less likely in sows than suckling piglets.

\section{Complete genomic characterisation of Sichuan PDCoV strains}

The complete genomes of four novel PDCoV strains, representative of epidemic strains, were sequenced and analysed to perform a molecular characterisation of PDCoV in Sichuan. The results showed that the complete genomic sequences of strains $\mathrm{CH} / \mathrm{SC} / 2017, \mathrm{CH} / \mathrm{SC} / 2018 / 1, \mathrm{CH} /$ $\mathrm{SC} / 2018$, and $\mathrm{CH} / \mathrm{SC} / 2019$ were $25,402 \mathrm{nt}, 25,413 \mathrm{nt}$, $25,414 \mathrm{nt}$, and 25,392 nt in length, respectively, and all had a typical PDCoV genome organisation. Sequence alignment analysis showed that the $\mathrm{CH} / \mathrm{SC} / 2017$ and $\mathrm{CH} /$ SC/2019 strains both had an 11-nt deletion in the 3'UTR region when compared with strains $\mathrm{CH} / \mathrm{SC} / 2018$ and $\mathrm{CH} /$ SC/2018/1 (Fig. 1). All four sequenced strains had a 6-nt deletion in the non-structural gene (ns) 2 region (ORF1ab), which has been observed in some other Chinese strains. Additionally, $\mathrm{CH} / \mathrm{SC} / 2019$ had a 9-nt deletion in the ns 3 region (ORF1ab) when compared with most Chinese strains. This deletion is also present in strain $\mathrm{CH} / \mathrm{Sichuan} / \mathrm{S} 27 / 2012$, detected previously in Sichuan, and in strains from Thailand 


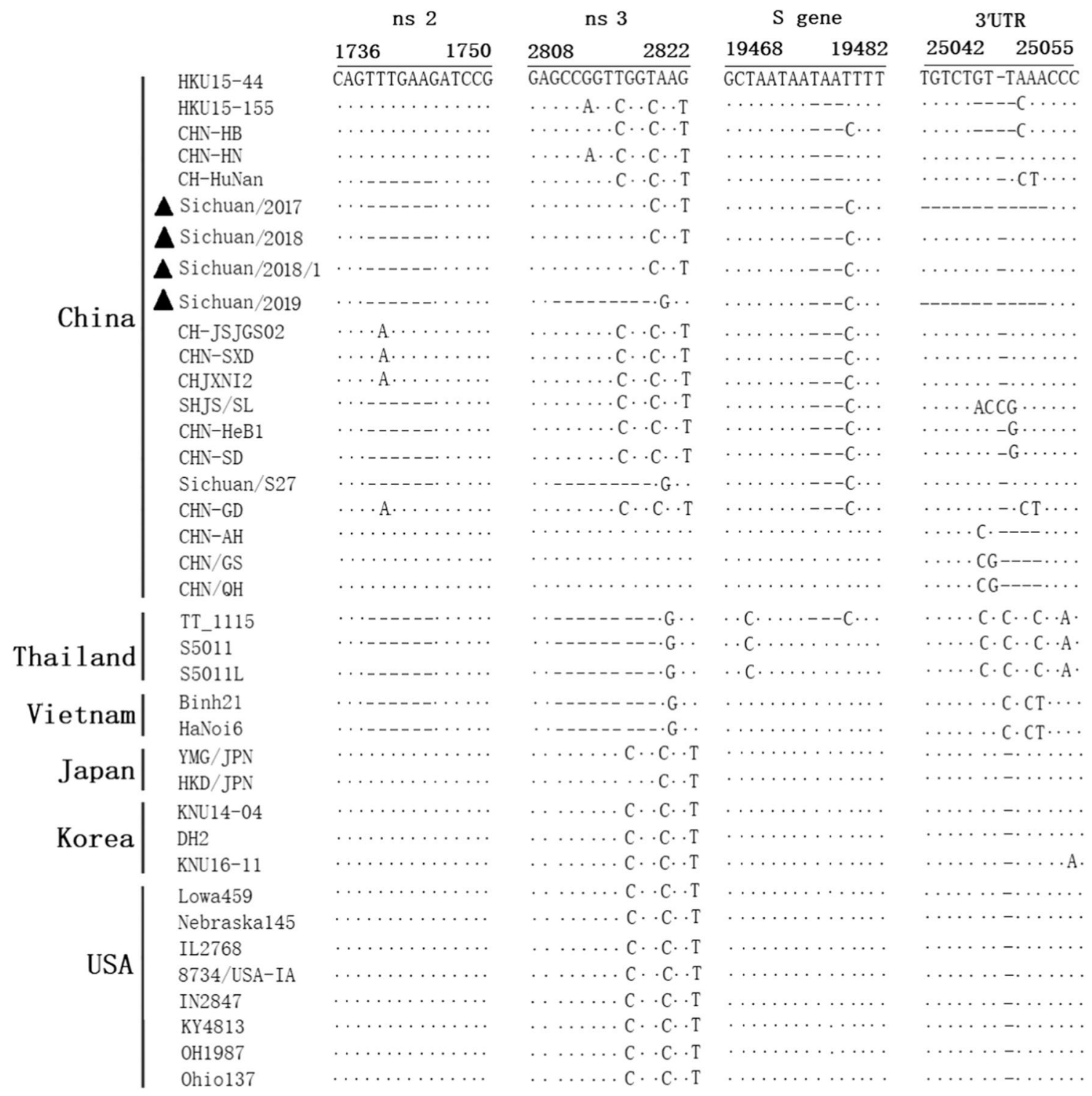

Fig. 1 The four main deletions or insertions in the complete genome sequence alignment. A multiple sequence alignment was constructed using ClustalW in the DNASTAR software. The four PDCoV strains sequenced in this study are indicated by black triangles. A dot $(\bullet)$

indicates that the nucleotide exactly matches the reference sequence. A dash (-) indicates that the nucleotide is deleted relative to the reference sequence

and Vietnam. Moreover, compared with strains from other countries, a 3-nt (TAA) deletion was observed in the S gene of the four novel strains, which is also present in most Chinese PDCoV strains, except early Chinese strains (CHN-GD and $\mathrm{CHN}-\mathrm{AH}$ ).

Pairwise nucleotide sequence comparisons showed that the four sequenced strains shared 98.9\%-99.3\% nucleotide sequence identity with each other, and shared $97.6 \%-99.0 \%$ nucleotide and $98.0 \%-99.5 \%$ aa sequence identity with reference PDCoV sequences. Interestingly, the full genomes of the $\mathrm{CH} / \mathrm{SC} / 2017, \mathrm{CH} / \mathrm{SC} / 2018$, and $\mathrm{CH} / \mathrm{SC} / 2018 / 1$ strains all had low nucleotide sequence identity $(97.6 \%)$ to strains

from Vietnam and Thailand, while the $\mathrm{CH} / \mathrm{SC} / 2019$ strain shared relatively high nucleotide sequence identity (98.9\%) with these strains, suggesting that the Sichuan PDCoV strains might have undergone genetic changes and continued to evolve.

\section{Phylogenetic analysis of PDCoV Sichuan strains}

Phylogenetic analysis was carried out using the whole-genome and $\mathrm{S}$ gene sequences of the four sequenced strains and other reference PDCoV strains (Fig. 2A and B. The phylogenies based on the complete genome sequences and the $\mathrm{S}$ gene both 


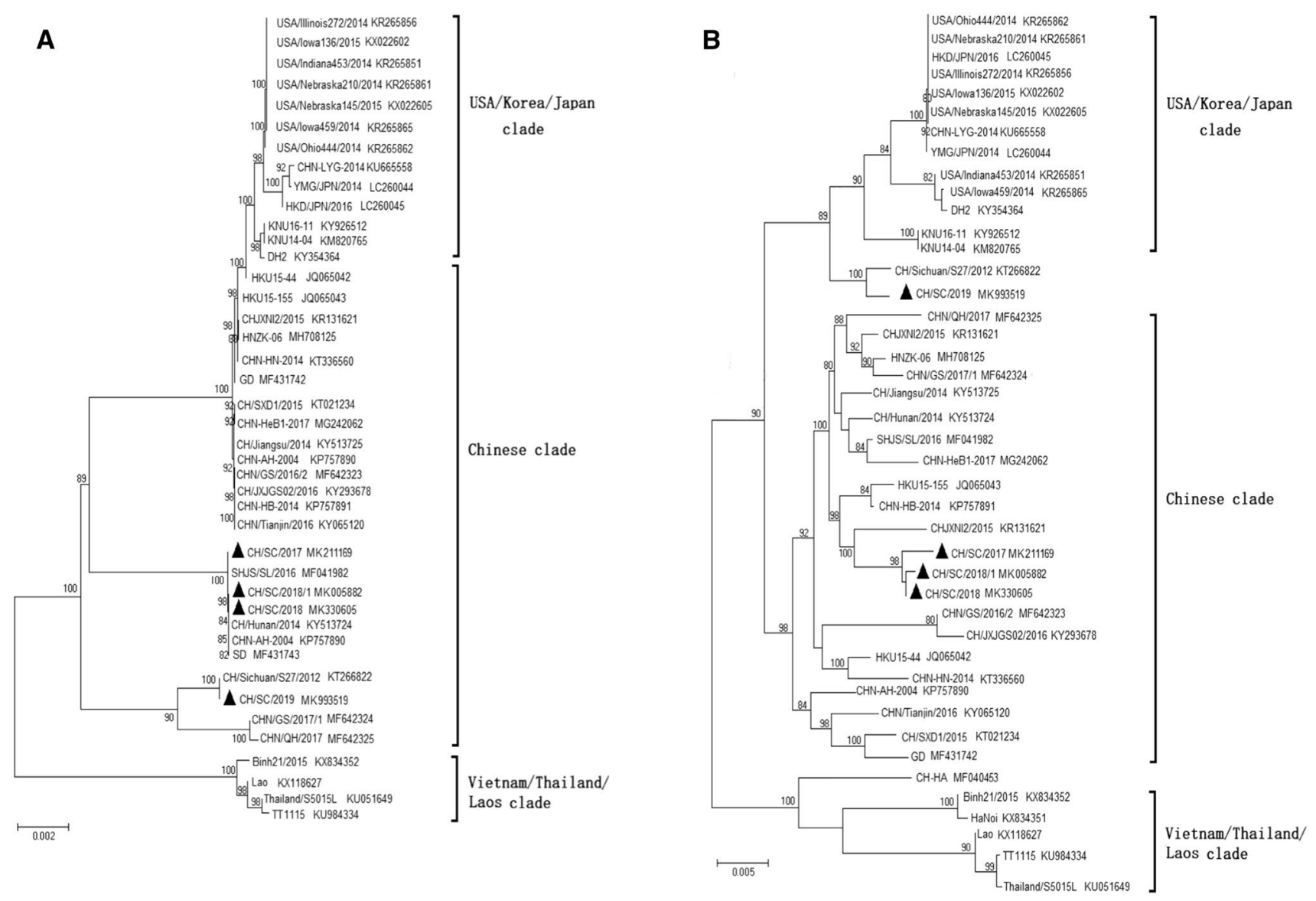

Fig. 2 Maximum-likelihood phylogenetic analysis based on (A) full-length genome sequences and (B) S gene sequences of PDCoV isolates. The scale bar indicates the number of nucleotide substitutions per site. The four strains sequenced in this study are indicated by black triangles

revealed that the PDCoV strains showed obvious regional characteristics, as has been reported previously [11]. Phylogenetic analysis based on whole genome sequences indicated that the PDCoV CH/SC/2017, CH/SC/ 2018/1, and CH/SC/ 2018 strains grouped within the Chinese clade and are more closely related to strains SHJS/SL/2016 and CH/HN/2014. However, the $\mathrm{CH} / \mathrm{SC} / 2019$ strain is located in another subcluster and is similar to the $\mathrm{CH} / \mathrm{QH} / 2017$ and $\mathrm{CH} / \mathrm{GS} / 2017$ strains, which were isolated from piglets in recent years, suggesting that the origin of these isolates may be different.

A phylogenetic tree based on $\mathrm{S}$ gene sequences revealed that $\mathrm{CH} / \mathrm{SC} / 2017, \mathrm{CH} / \mathrm{SC} / 2018 / 1$, and $\mathrm{CH} / \mathrm{SC} / 2018$ belong to the same group, while the $\mathrm{CH} / \mathrm{SC} / 2019$ strain clustered in a large clade with strains from the USA, Japan, and Korea and demonstrated genetic differences when compared to the other Sichuan strains.

\section{Discussion}

In this study, RT-PCR analysis of 634 clinical samples from pigs with diarrhoea collected from 13 regions in Sichuan province from January 2017 and June 2019 revealed a positive rate of PDCoV of $13.25 \%$, while that of PEDV was $32.18 \%$, indicating that PEDV is still the primary pathogen causing porcine diarrhoea, which is consistent with previous studies [12]. The prevalence of PDCoV in diarrhoeal pigs in Sichuan was somewhat lower than in other provinces, such as Henan (23.49\%) and Guangdong (21.8\%), but a molecular-based detection study showed a similar low prevalence of PDCoV infection in Jiangsu (4.94\%) and Hubei (6.45\%) [13]. The prevalence of PDCoV in Qinghai and Tibet is also low, with a 
positive rate of only $3.70 \%$ [14]. These data show that the prevalence of PDCoV in diarrhoeal pigs differs among the Chinese provinces, and more information regarding the molecular epidemiology of PDCoV in China is needed. In this study, the number of samples collected and regions investigated was limited, and therefore more samples need to be collected and tested, and PDCoV surveillance should be continued. Also, since the aim of this study was only to monitor the prevalence of PDCoV in diarrhoeal pigs more samples, including those from pigs without diarrhoea, are needed to evaluate the prevalence of PDCoV in Sichuan pig herds.

Although the overall prevalence of PDCoV in this study was relatively low, $\mathrm{PDCoV}$ was detected in most regions of Sichuan, implying that PDCoV prevention measures have not been very effective, and further study is needed to prevent and control this novel enteric virus. Statistical analysis demonstrated a higher chance of PDCoV infection in winter and spring, which is consistent with previous studies [15], suggesting that we should pay more attention to preventing infections during these seasons. Furthermore, we detected PDCoV in sows, as described previously [16]. However, unlike a previous study, our results showed that suckling piglets had a 2.77 times higher risk of PDCoV infection than sows. Previous reports have suggested that older pigs are more susceptible to PDCoV than piglets, which was not confirmed by our results [17]. Therefore, more clinical samples from older pigs are needed to test this conclusion. We plan to continue to monitor PDCoV infections in sows. Moreover, it has been reported that PDCoV/PEDV coinfection is more common in Chinese pigs than PDCoV/porcine rotavirus coinfection in US pig herds $[18,19]$. Our detection data are consistent with this, also showing that suckling piglets have a higher chance of PDCoV/PEDV coinfection.

The results of sequence analysis showed that the four strains had some unique deletions/insertions compared to other PDCoV strains. The $\mathrm{CH} / \mathrm{SC} / 2019$ strain, like the $\mathrm{CH} /$ Sichuan/S27/2012 strain, had a 6-nt deletion in the ns 2 region and a 9-nt deletion in the ns 3 region, respectively, while the others strains only had a 6-nt deletion in ns 2 . Moreover, four stains had a 3-nt deletion in the deletion of the $\mathrm{S}$ gene, resulting in one amino acid. This deletion exists in most Chinese PDCoV isolates. The $\mathrm{S} 1$ region is responsible for virus receptor binding [20], suggesting that this aa deletion may have some biological significance regarding viral replication or pathogenesis. Importantly, the $\mathrm{CH} / \mathrm{SC} / 2017$ and $\mathrm{CH} / \mathrm{SC} / 2019$ strains had a unique 11-nt deletion in the $3^{\prime} \mathrm{UTR}$ region. This is the first report of a PDCoV strain with such a deletion. However, we do not know whether these naturally occurring deletions or insertions have biological significance in PDCoV biology and pathogenesis. The four sequenced PDCoV strains were all collected from suckling piglets, but the clinical signs in these piglets were different. The piglets infected with the $\mathrm{CH} / \mathrm{SC} / 2017$ and $\mathrm{CH} / \mathrm{SC} / 2019$ strains suffered from severe watery diarrhoea and died after three days of diarrhoea, while the suckling piglet with the $\mathrm{CH} / \mathrm{SC} / 2018$ and $\mathrm{CH} /$ SC/2018/1 strains had moderate diarrhoea and then recovered. Were these different clinical signs associated with the 11-nt deletion in the $3^{\prime} \mathrm{UTR}$ or another deletion/insertion? We will continue to isolate PDCoV strains for further investigation.

A phylogenetic tree constructed based on complete genome sequences indicated that these strains might have evolved from different Chinese strains. In the phylogenetic tree based on the $\mathrm{S}$ gene, the Sichuan strains clustered in a different clade. The $\mathrm{CH} / \mathrm{SC} / 2017, \mathrm{CH} / \mathrm{SC} / 2018 / 1$, and $\mathrm{CH} /$ $\mathrm{SC} / 2018$ strains are in the China lineage, like most Chinese PDCoVs, while the $\mathrm{CH} / \mathrm{SC} / 2019$ strain is in the USA/Japan/ South Korea lineage. This is possibly due to recombination or separate evolution Additional PDCoV sequences are needed to identify possible recombination and evolution events.

In summary, we report for the first time the prevalence and genetic properties of PDCoV in diarrhoeal pigs in Sichuan, which could provide further insights into the epidemiology and evolution of PDCoV in China.

Acknowledgements This research was supported by the Science and Technology Program of Sichuan Province: Development of Bivalent Live Vaccine for Porcine Epidemic Diarrhea and Porcine Pseudorabies (2017NZ0038), the National Key Research Program During the $13^{\text {th }}$ Five-Year Plan Period of China: Integrated Prevention and Control Technology for Major Diseases of Commercial Pigs and Integration of Safe Drug Use Technology (2018YFD051102), and the Program Sichuan Veterinary Medicine and Drug Innovation Group of China Agricultural Research System (CARS-SVDIP).

\section{Compliance with ethical standards}

Conflict of interest The authors declare that they have no conflict of interest.

Ethical approval This project was approved by the appropriate ethical review committee. The "Guidelines for Experimental Animals" of the Ministry of Science and Technology (Beijing, China) were followed.

\section{References}

1. Jung K, Hu H, Bryan E, Lu Z, Chepngeno J, Saif L (2015) Pathogenicity of 2 porcine deltacoronavirus strains in gnotobiotic pigs. Emerg Infect Dis 21(4):650-654

2. Hu H, Jung K, Vlasova N, Chepngeno J, Lu Z, Wang Q, Saif J (2015) Isolation and characterisation of porcine deltacoronavirus from pigs with diarrhea in the United States. J Clin Microbiol 53(3):1537-1548

3. Shang J, Zheng Y, Yang Y, Liu C, Geng Q, Tai W, Du L, Zhou Y, Zhang W, Li F (2017) Cryo-EM structure of porcine delta coronavirus spike protein in the pre-fusion state. J Virol 92(4):e01556-17 
4. Woo C, Lau K, Lam S, Lau C, Tsang K, Lau H, Bai R, Teng L, Tsang C, Wang M, Zheng J, Chan H, Yuen Y (2012) Discovery of seven novel mammalian and avian coronaviruses in the genus deltacoronavirus supports bat coronaviruses as the gene source of alphacoronavirus and betacoronavirus and avian coronaviruses as the gene source of gammacoronavirus and deltacoronavirus. $\mathbf{J}$ Virol 86(7):3995-4008

5. Marthaler D, Raymond L, Jiang Y, Collins J, Rossow K, Rovira A (2014) Rapid detection, complete genome sequencing, and phylogenetic analysis of porcine deltacoronavirus. Emerg Infect Dis 20(8):1347-1350

6. Ajayi T, Dara R, Misener M, Pasma T, Moser L, Poljak Z (2018) Herd-level prevalence and incidence of porcine epidemic diarrhoea virus (PEDV) and porcine deltacoronavirus (PDCoV) in swine herds in Ontario, Canada. Transbound Emerg Dis 65(5):1197-1207

7. Lee S, Lee C (2014) Complete genome characterization of Korean porcine deltacoronavirus strain KOR/KNU14-04/2014. Genome Announc 2(6): $\mathrm{e}$ 01191-14

8. Liu J, Zuo Z, Gu Y, Luo X, Shi K, Hou S, Zhong F, Fan H (2018) Isolation and phylogenetic analysis of porcine deltacoronavirus from pigs with diarrhoea in Hebei province, China. Transbound Emerg Dis 65(3):874-882

9. Saeng-Chuto K, Stott J, Wegner M, Senasuthum R, Tantituvanont A, Nilubol D (2017) Retrospective investigation and evolutionary analysis of a novel porcine deltacoronavirus strain detected in Thailand from 2008 to 2015. Arch Virol 162(7):2103-2108

10. Wang L, Byrum B, Zhang Y (2014) Detection and genetic characterization of deltacoronavirus in pigs, Ohio, USA. Emerg Infect Dis 20(7):1227-1230

11. Zhang J (2016) Porcine Deltacoronavirus: Overview of infection dynamics, diagnostic methods, prevalence and genetic evolution. Virus Res 226(2):71-84

12. Jang G, Lee K, Kim H, Lee C (2017) Prevalence, complete genome sequencing and phylogenetic analysis of porcine deltacoronavirus in South Korea, 2014-2016. Transbound Emerg Dis 64(5):1364-1370

13. Dong N, Fang L, Zeng S, Sun Q, Chen H, Xiao S (2015) Porcine deltacoronavirus in mainland China. Emerg Infect Dis 21(12):2254-2255
14. Wang M, Wang Y, Baloch R, Pan Y, Tian L, Xu F, Shivaramu S, Chen S, Zeng Q (2018) Detection and genetic characterization of porcine deltacoronavirus in Tibetan pigs surrounding the QinghaiTibet Plateau of China. Transbound Emer Dis 65(2):363-369

15. Zhang HL, Liang QQ, Li BX, Cui XG, Wei XL, Ding QW, Wang YB, Hu H (2019) Prevalence, phylogenetic and evolutionary analysis of porcine deltacoronavirus in Henan province, China. Prev Vet Med 166:8-15

16. Mai K, Feng J, Chen G, Li D, Zhou L, Bai Y, Wu Q, Ma J (2018) The detection and phylogenetic analysis of porcine deltacoronavirus from Guangdong Province in Southern China. Transbound Emerg Dis 65(1):166-173

17. Suzuki T, Shibahara T, Imai N, Yamamoto T, Ohashi S (2018) Genetic characterization and pathogenicity of Japanese porcine deltacoronavirus. Infect Genet and Evol 61:176-182

18. Song D, Zhou X, Peng Q, Chen Y, Zhang F, Huang T, Zhang T, Li A, Huang D, Wu Q, He H, Tang Y (2015) Newly emerged porcine deltacoronavirus associated with diarrhoea in swine in China: identification, prevalence and full-length genome sequence analysis. Transbound Emerg Dis. 62(6):575-580

19. Marthaler D, Raymond L, Jiang Y, Collins J, Rossow K, Rovira A (2014) Rapid detection, complete genome sequencing, and phylogenetic analysis of porcine Deltacoronavirus. Emerg Infect Dis 20:1347-1349

20. Li W, Hulswit G, Kenney P, Widjaja I, Jung K, Alhamo A, Dieren B, Kuppeveld M, Saif J, Bosch J (2018) Broad receptor engagement of an emerging global coronavirus may potentiate its diverse cross-species transmissibility. Proc Natl Acad Sci USA 115(22):E5135-E5143

Publisher's Note Springer Nature remains neutral with regard to jurisdictional claims in published maps and institutional affiliations. 